\title{
State-Dependent Spike and Local Field Synchronization between Motor Cortex and Substantia Nigra in Hemiparkinsonian Rats
}

\author{
Elena Brazhnik, Ana V. Cruz, Irene Avila, Marian I. Wahba, Nikolay Novikov, Neda M. Ilieva, Alex J. McCoy, \\ Colin Gerber, and Judith. R. Walters \\ Neurophysiological Pharmacology Section, National Institute of Neurological Disorders and Stroke, National Institutes of Health, Bethesda, Maryland \\ 20892-3702
}

Excessive beta frequency oscillatory and synchronized activity has been reported in the basal ganglia of parkinsonian patients and animal models of the disease. To gain insight into processes underlying this activity, this study explores relationships between oscillatory activity in motor cortex and basal ganglia output in behaving rats after dopamine cell lesion. During inattentive rest, $7 \mathrm{~d}$ after lesion, increases in motor cortex-substantia nigra pars reticulata (SNpr) coherence emerged in the 8-25 Hz range, with significant increases in local field potential (LFP) power in SNpr but not motor cortex. In contrast, during treadmill walking, marked increases in both motor cortex and SNpr LFP power, as well as coherence, emerged in the $25-40 \mathrm{~Hz}$ band with a peak frequency at $30-35 \mathrm{~Hz}$. Spike-triggered waveform averages showed that $77 \%$ of SNpr neurons, $77 \%$ of putative cortical interneurons, and $44 \%$ of putative pyramidal neurons were significantly phase-locked to the increased cortical LFP activity in the $25-40 \mathrm{~Hz}$ range. Although the mean lag between cortical and SNpr LFPs fluctuated around zero, SNpr neurons phase-locked to cortical LFP oscillations fired, on average, $17 \mathrm{~ms}$ after synchronized spiking in motor cortex. High coherence between LFP oscillations in cortex and SNpr supports the view that cortical activity facilitates entrainment and synchronization of activity in basal ganglia after loss of dopamine. However, the dramatic increases in cortical power and relative timing of phase-locked spiking in these areas suggest that additional processes help shape the frequency-specific tuning of the basal ganglia-thalamocortical network during ongoing motor activity.

\section{Introduction}

Excessive synchronization of neuronal activity in the $12-35 \mathrm{~Hz}$ frequency range has been reported in the subthalamic nucleus (STN) and globus pallidus pars interna of Parkinson's disease (PD) patients undergoing implantation of deep brain stimulation electrodes. This activity is reduced by L-dopa administration and is thought to produce the akinesia and bradykinesia characteristic of PD (Brown et al., 2001; Levy et al., 2002; Brown, 2003; Priori et al., 2004; Alonso-Frech et al., 2006; Weinberger et al., 2006; Kühn et al., 2009).

Animal models of PD provide an opportunity to investigate mechanisms underlying the emergence of synchronized and oscillatory activity in the basal ganglia after loss of dopamine. Stud-

Received Feb. 27, 2012; revised April 13, 2012; accepted April 22, 2012.

Author contributions: E.B., I.A., and J.R.W. designed research; E.B., I.A., and N.N. performed research; E.B., A.V.C., I.A., M.I.W., N.M.I., A.J.M., C.G., and J.R.W. analyzed data; E.B., A.V.C., I.A., and J.R.W. wrote the paper.

This work was supported by the Intramural Research Program of the NINDS, NIH. We thank Tom Talbot, Daryl Bandy, and Newlin Morgan in the Section on Instrumentation, NIMH/NINDS for design and fabrication of the rotary treadmill.

The authors declare no competing financial interests.

Correspondence should be addressed to Dr. Judith R. Walters, Neurophysiological Pharmacology Section, NINDS, NIH, 35 Convent Drive, Building 35, Room 1C905, Bethesda, MD 20892-3702. E-mail:waltersj@ninds.nih.gov.

I. Avila's present address: Neural Circuits and Cognition Unit, NIA, NIH, BG BRC RM 09C220, 251 Bayview Boulevard, Baltimore, MD 21224-6825.

DOI:10.1523/JNEUROSCI.0943-12.2012

Copyright $\odot 2012$ the authors $\quad 0270-6474 / 12 / 327869-12 \$ 15.00 / 0$ ies in the anesthetized hemiparkinsonian rat have shown that dopamine depletion is associated with changes in striatal processing of cortical input that promote transmission of the dominant $1 \mathrm{~Hz}$ cortical rhythm to downstream basal ganglia nuclei (Tseng et al., 2001; Murer et al., 2002; Mallet et al., 2006; Walters et al., 2007; Zold et al., 2011). Additional in vivo and in vitro studies suggest that increases in the sensitivity of the STN to cortical input from the hyperdirect pathway and interactions between the STN and globus pallidus pars externa (GPe) facilitate the emergence of beta range activity in the basal ganglia after loss of dopamine (Baufreton et al., 2005; Shen and Johnson, 2005; Mallet et al., 2008a; Holgado et al., 2010; Cruz et al., 2011). Collectively, these observations plus evidence of increased coherence between the STN and cortex after dopamine cell lesion in awake behaving rats (Sharott et al., 2005; Mallet et al., 2008b; Degos et al., 2009) and PD patients (Williams et al., 2002; Fogelson et al., 2006) support the hypothesis that a notable consequence of dopamine cell loss is the enhanced tendency for basal ganglia activity to become entrained by oscillatory activity expressed in the cortex (Murer et al., 2002).

The suggestion that cortical rhythms contribute to the emergence of synchronized activity in basal ganglia output after dopamine cell death raises a number of questions. Does basal ganglia activity become indiscriminately entrained by whatever cortex rhythms are dominant after loss of dopamine, or preferentially entrained by cortical output within specific frequency ranges that 
promote resonance in basal ganglia subcircuits and/or in basal ganglia-thalamocortical circuits? A further issue is the extent to which synchronized activity in the cortex is itself altered after dopamine loss. Changes could result from direct effects of dopamine loss or compensatory response to motor symptoms, as well as feedback from basal ganglia output. The present study sought to investigate these questions by determining whether statedependent changes in basal ganglia output (Avila et al., 2010) are correlated with changes in motor cortex activity after dopamine cell lesion. Simultaneous recordings of local field potential (LFP) and spiking activity were obtained from motor cortex layers 5/6 and substantia nigra pars reticulata ( $\mathrm{SNpr}$ ) during inattentive rest and treadmill walking in hemiparkinsonian and control rats. An additional goal was to assess temporal relationships between LFP and spiking activity in cortex and SNpr during periods of peak coherence. L-Dopa and dopamine receptor antagonist treatments were used to further probe correlations between loss of dopamine, oscillatory activity, and motor cortex-SNpr coherence.

\section{Materials and Methods}

All experimental procedures were conducted in accordance with the NIH Guide for Care and Use of Laboratory Animals and approved by the NINDS Animal Care and Use Committee. Every effort was made to minimize the number of animals used and their discomfort.

\section{Surgical procedures}

Male Long-Evans rats (Taconic Farm), 250-300 g, were housed on a 12:12 h reversed light cycle. Rats were anesthetized intraperitoneally with $75 \mathrm{mg} / \mathrm{kg}$ ketamine and $0.25 \mathrm{mg} / \mathrm{kg}$ dexmedetomidine before surgery for implantation of chronic recording electrodes. The incision area was shaved and a long-acting local anesthetic ( $1 \%$ mepivacaine $\mathrm{HCl}$ solution) injected along the intended incision lines. Ophthalmic ointment was applied to prevent corneal dehydration and lidocaine gel placed in the ear canals. Rats were placed in a stereotaxic frame (David Kopf Instruments) fitted with atraumatic ear bars with their skull leveled in the dorsalventral plane. A heating pad was used to maintain body temperature at $\sim 37^{\circ} \mathrm{C}$.

All rats had electrode bundles implanted unilaterally in the motor cortex layers 5/6 and in the SNpr. One set of 10 rats received local injections of 6-hydroxydopamine (6-OHDA) in the medial forebrain bundle to lesion dopamine cells in the substantia nigra pars compacta at the time the electrode bundles were implanted. Another set of 8 rats had a chronic indwelling cannula implanted unilaterally over the area of the medial forebrain bundle at the time electrode bundles were implanted in the motor cortex and SNpr. These rats served as nonlesioned controls initially and at day 8 after cannulation, the cannula was used for injection of 6-OHDA. Data from seven of these rats were used postlesion, in addition to six from the original group of lesioned rats for cortex-SNpr spike-LFP temporal relationship data.

Electrode bundles consisted of eight stainless steel insulated microwires with an additional ninth wire $(\sim 1 \mathrm{~mm}$ scraped tip $)$ that served as a local reference. Bundles had diameters of $\sim 350 \mu \mathrm{m}$ (NB Labs). Holes were drilled over the target coordinates for the SNpr (anterior: $+3.2 \mathrm{~mm}$ from the lambdoid suture; lateral: $2.2 \mathrm{~mm}$ from the sagittal suture; ventral: $-8.0 \mathrm{~mm}$ from the skull surface) and for the motor cortex (anterior: $+2.0 \mathrm{~mm}$ from bregma; lateral: $2.5 \mathrm{~mm}$; ventral: -2.0 $\mathrm{mm})$. Ground wires from each bundle were connected to a screw located above the cerebellum. In most rats, electromyogram (EMG) electrodes were implanted bilaterally into the shoulder (scapularis) muscles, as described previously (Avila et al., 2010).

To protect noradrenergic neurons, 6-OHDA injection was preceded by administration of $15 \mathrm{mg} / \mathrm{kg}$ desmethylimipramine (i.p.). Standard stereotaxic procedures were used to target the left medial forebrain bundle (anterior: $+4.4 \mathrm{~mm}$ from the lambdoid suture; lateral: $+1.2 \mathrm{~mm}$ from the sagittal suture; ventral: $-8.3 \mathrm{~mm}$ from the skull surface). Six micrograms of 6-OHDA hydrobromide in $3 \mu \mathrm{l}$ of $0.9 \%$ saline with $0.01 \%$ ascorbic acid was infused via a 27 gauge stainless steel cannula into the medial forebrain bundle at a rate of $1 \mu \mathrm{l} / \mathrm{min}$ over $3 \mathrm{~min}$ via a syringe pump (Harvard Apparatus). The cannula remained at the target site for 3 min after the infusion was completed to prevent diffusion of the neurotoxin.

\section{Behavior}

A circular treadmill was used during recordings to control the behavior of the rats when walking. Before the surgical procedures, rats were trained to walk in both directions on the circular treadmill at a speed of 9 RPMs. Postlesion, rats were gradually reexposed to the treadmill during the first week and examined for their ability to walk in both directions. As previously reported, the speed and design were adjusted so that both control and lesioned rats were capable of performing on the treadmill, so that after unilateral dopamine cell lesions, the hemiparkinsonian rats could make reasonable progress on the circular treadmill if they were oriented in the direction ipsiversive to the unilateral lesion, with their affected paws on the outside of the circular path (Avila et al., 2010). If they were oriented in the opposite direction, contraversive to the lesion, they had considerable difficulty walking, and generally froze or reared and tried to turn around. Lesion efficacy was also assessed by step testing (Olsson et al., 1995) during the first week postlesion. One week postlesion, when spiking activity and LFP recordings were initiated, sessions were videotaped to provide further confirmation of the level of motor activity in the rats.

\section{Electrophysiological recordings}

At two time periods after surgery, days 7-9 and days 21-24 (referred to as day 7 and day 21 in Results, below), spike trains, LFP activities, and EMG signals from the scapularis muscles were recorded during periods of rest or treadmill walking. Recordings were obtained during 5 min epochs of walking in the direction ipsiversive to the lesion or cannula implantation, followed by $5-10 \mathrm{~min}$ of rest with the treadmill turned off. Epochs were identified as inattentive rest when rats were resting with their eyes open and flat EMG activity was observed in the scapularis muscle.

Extracellular spike trains, LFPs and bipolar EMGs were amplified and filtered using Plexon and Spike2 (CED) systems. Recordings from each of the eight microwire electrode bundles were referenced to their own scraped ninth wire. Spikes were amplified with a gain in the range of $5000-15,000 \times$, digitized at $40 \mathrm{kHz}$, and bandpass filtered at $154-8800$ $\mathrm{Hz}$. LFP recordings were amplified by $1000 \times$, sampled at $1 \mathrm{kHz}$, and filtered at 3-170 Hz. EMG recordings were amplified by $1000 \times$, digitized at $1 \mathrm{kHz}$, and filtered at $0.1-1 \mathrm{kHz}$. Single units were sorted off-line based on clustering and principal component analysis using Spike2 software (see Data analysis, below).

\section{Drug treatments}

Twenty-one to $24 \mathrm{~d}$ postlesion, after baseline ipsiversive walking, rats received a low dose of L-dopa ( $5 \mathrm{mg} / \mathrm{kg}$ with $15 \mathrm{mg} / \mathrm{kg}$ benserazide, i.p.; Sigma-Aldrich) and 40-50 min later, a dose of eticlopride $(0.2 \mathrm{mg} / \mathrm{kg}$; Sigma-Aldrich).

\section{Data analysis}

LFP and extracellular unit activity were analyzed using custom-written Spike2 and MATLAB scripts. Epochs of $60 \mathrm{~s}$ representative of the overall recording and free of major artifacts were used to calculate LFP power during inattentive rest and walk periods. For each rat, data from at least two electrodes per brain region and two epochs per behavioral state were used for power, coherence, peak frequency, and spike-triggered waveform averages (STWA). Since rats with left hemispheric lesions experienced difficulty walking contraversive to the lesion, data were taken only when rats were walking in the ipsiversive direction.

Spectral analysis of LFPs. Power and coherence were assessed over the range of $8-50 \mathrm{~Hz}$. For fast Fourier transfer (FFT)-based analysis of power and coherence, LFP recordings were smoothed to $500 \mathrm{~Hz}$ and LFP power spectra and coherence $(\sim 1 \mathrm{~Hz}$ resolution) were obtained using a Spike2 script. Changes in power and coherence over specific frequency ranges were analyzed using two-way ANOVA and one-way and two-way repeated-measures (RM) ANOVA with post hoc comparisons with the level of significance $p<0.05$. 
Peak frequencies in power and coherence spectra were identified as significant if they met three criteria. First, the peak frequency amplitudes were larger than those in the preceding and following eight $1 \mathrm{~Hz}$ frequency bins (Doyle et al., 2005; Alonso-Frech et al., 2006). Second, power spectrum slope changed sign from positive before the peak to negative after the peak. Third, data surrounding the maximum had a downward concavity according to second derivative analysis.

To visualize spectral power changes over time for the selected epochs, time-frequency wavelet spectra were constructed using continuous wavelet transforms. The Morlet wavelet was applied to the LFPs using 128 frequency scales and a time resolution of $\sim 750 \mathrm{~ms}$ (Time-Frequency Toolbox; http:tftb.nongnu.org). Wavelet spectra were scaled to best show the high beta activity against minimum background noise. Coherence spectra were constructed using an FFT-based analysis with a $10 \mathrm{~s}$ moving window (Chronux; http://chronux.org).

Analysis of LFP directionality and amplitude envelopes. To assess phase and lag times between the oscillatory activity of motor cortex and SNpr in the $25-40 \mathrm{~Hz}$ frequency range, the cross-correlations between the LFPs and their envelopes (slow time amplitude fluctuations) recorded from both regions (Adhikari et al., 2010) were analyzed. The analysis was performed using custom-written Matlab (Mathworks) programs. The signals were filtered by a conventional bandpass finite impulse response (FIR) filter based on a Hamming window. The LFP envelope was calculated as the magnitude of the Hilbert transform of the filtered signal, as computed with the Matlab hilbert function. The cross-correlations between the LFP signals and the envelopes were calculated by means of the Matlab function xcorr (Signal Processing Toolbox). We adopted the convention that positive lags indicate motor cortex signal precedes the SNpr signal.

Cell sorting and motor cortex cell type classification. Spikes from motor cortex and SNpr recordings were sorted using principal components analysis (PCA) (Spike2). The durations of well isolated spike waveforms from spike trains recorded from motor cortex were measured to categorize neurons as either putative pyramidal neurons or interneurons. The time between the trough of each action potential and the subsequent peak was measured. Using $k$-means cluster analysis, the action potential widths of spikes were classified into two different distributions, with the shorter duration group including action potential widths in the $0.1-0.3$ $\mathrm{ms}$ range and the longer group exceeding $0.38 \mathrm{~ms}$. Only spike trains with average firing rates $\geq 2 \mathrm{~Hz}$ were included. Although a variety of approaches have been applied to measure cortical action potential duration, studies consistently report a bimodal distribution of action potential widths. A number of studies comparing electrophysiological characteristics with neuron morphology in the cortex have reported that two neuronal subpopulations can be reliably distinguished based on the duration of their extracellularly recorded action potential, with pyramidal neuron durations being longer than interneuron durations (Wilson et al., 1994; Barthó et al., 2004; Tierney et al., 2004; Parr-Brownlie and Hyland, 2005; Tseng et al., 2006; Mitchell et al., 2007; Isomura et al., 2009). Based on the bimodal distribution of the present data, the criterion dividing putative pyramidal and interneurons was set at $0.30 \mathrm{~ms}$. The majority (90/134, $67 \%$ of recorded neurons) had action potential durations $>0.38 \mathrm{~ms}$ and were classified as putative pyramidal neurons. In this paper, the terms pyramidal neuron and interneuron refer to putative pyramidal and putative interneurons, respectively.

Spike-triggered waveform analysis. To assess the temporal relationship between the spiking activity of individual neurons and LFP oscillations, spike timing and phase with respect to spike-triggered LFP averages were quantified during $200 \mathrm{~s}$ of treadmill walking for spike trains with average firing rates $\geq 2 \mathrm{~Hz}$. LFPs were bandpass filtered (25-40 Hz and 12-18 $\mathrm{Hz}$ ) using an FIR filter (Spike2). Peak-to-trough amplitudes of the STWAs at or around the spike (zero time) were obtained as a measure of phase locking of spike train to the dominant LFP oscillation. Each spike train was then shuffled 20 times and each shuffled spike train was used to create an STWA. The peak-to-trough values of the shuffled STWAs were normally distributed. Spikes were considered to be significantly correlated with the LFP oscillations when the peak-to-trough amplitude of the unshuffled spike train STWA was greater than 3 SDs of the mean of the shuffled distribution.
Data from the spike-LFP analysis were processed in three ways. First, the percentage of spike trains that were significantly correlated with LFP oscillations was calculated for different neuronal subtypes in depleted and control animals. Second, to assess the overall extent of phase-locking of cortical pyramidal neurons, interneurons, and SNpr neurons in the control versus lesioned rats, mean ratios of unscrambled/scrambled peak-to-trough amplitudes for spike trains were obtained. Third, the phase of the spikes with respect to the peaks of the LFP oscillations was calculated from the STWAs. Spikes that occurred in the peak or trough of the LFP are at $0^{\circ}$ or $180^{\circ}$ phase, respectively.

Unless stated otherwise, all results in this manuscript are presented as the mean value \pm SEM.

\section{Histology}

Rats were deeply anesthetized and recording sites were marked by electrolytic lesions. Rats were perfused intracardially with $200 \mathrm{ml}$ of cold saline followed by $200 \mathrm{ml}$ of $4 \%$ paraformaldehyde in PBS. Fixed brains were sliced and $40 \mu \mathrm{m}$ coronal sections containing the substantia nigra pars compacta were immunostained for tyrosine hydroxylase (TH; primary rabbit polyclonal anti-TH antibody, 1:200 dilution; Pel-Freez Biologicals; and biotinylated anti-rabbit IgG secondary antibody, 1:200 dilution; Vector Labs). The staining was processed using avidin-biotin-perioxidase complex (ABC kit; Vector Labs) and 0.05\% 3,3'-diaminobenzidine tetrahydrochloride with $0.01 \% \mathrm{H}_{2} \mathrm{O}_{2}$ (DAB kit; Vector Labs) until intense brown color emerged. ImageJ software (NIH) was used to evaluate the extent of tyrosine hydroxylase staining in anterior, middle, and posterior sections of the substantia nigra and ventral tegmental area. Images were first converted to grayscale and then tyrosine hydroxylase-stained cell bodies and fibers were thresholded in the substantia nigra of the intact hemisphere. Identical settings were applied to the lesioned hemisphere. Data showed severe loss of tyrosine hydroxylase-stained neurons and fibers in the substantia nigra of the dopamine-depleted hemisphere with relative density after thresholding averaging $<10 \%$ of that in the contralateral substantia nigra of the nonlesioned hemisphere. Tyrosine hydroxylase staining also showed partial loss of dopamine neurons and fibers in the lateral ventral tegmental area in the dopamine-lesioned hemisphere. To verify electrode bundle placement, recording sites were marked by the electrolytic lesion and sections were counterstained with cresyl violet and 5\% potassium ferricyanide $/ 9 \% \mathrm{HCl}$ to reveal iron disposition marking the location of the electrode tips.

\section{Results}

Oscillatory LFP activity in the SNpr after dopamine cell lesion Recordings from chronically implanted electrodes in the SNpr confirmed previous observations (Avila et al., 2010) of increases in oscillatory LFP activity in this nucleus in the dopaminedeprived hemisphere 1 week after unilateral 6-OHDA-induced medial forebrain bundle dopamine cell lesion. As shown in wavelet-based scalograms (Fig. 1C,D) and FFT-based power spectra (Fig. $1 \mathrm{I}, \mathrm{J}$ ), these recordings also confirmed that dopamine lesion-induced changes in SNpr LFP power observed during treadmill walking have a different profile from those observed during inattentive rest.

During epochs of inattentive rest, SNpr LFP spectral power was significantly increased throughout the $8-25 \mathrm{~Hz}$ alpha/low beta frequency range, day 7 and 21 after dopamine cell lesion, with increases most prominent at the lower end of that range (Fig. $1 C, D, I)$. SNpr LFP power also appeared increased in frequencies $<8 \mathrm{~Hz}$ in the lesioned hemisphere of the hemiparkinsonian rats during rest; however, data analysis was not extended to the lower frequency ranges in the present study as the high-pass filters in the preamplifier limited accuracy below $\sim 5 \mathrm{~Hz}$. During rest epochs, no significant differences in SNpr LFP power were observed between control and dopamine-depleted hemispheres in the 25-50 Hz high beta/gamma range (Fig. $1 I$ ).

Continuous recordings from the unilaterally lesioned rats during epochs of treadmill walking were facilitated by the use of a circular treadmill; the rats were able to make regular progress 


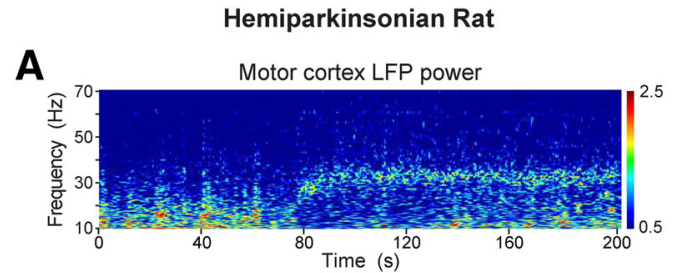

C

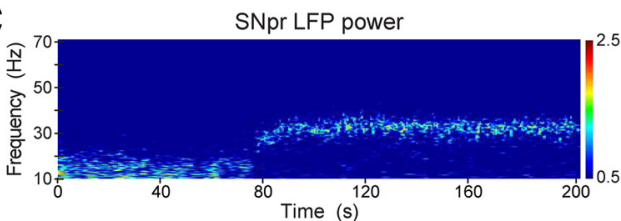

$\mathbf{E}$

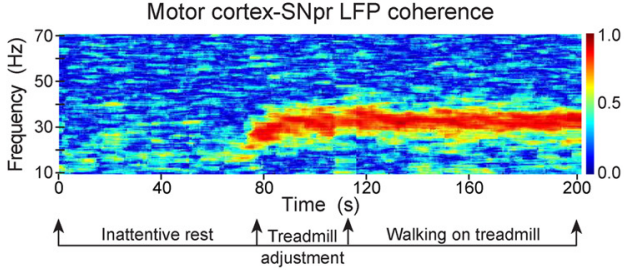

Inattentive Rest
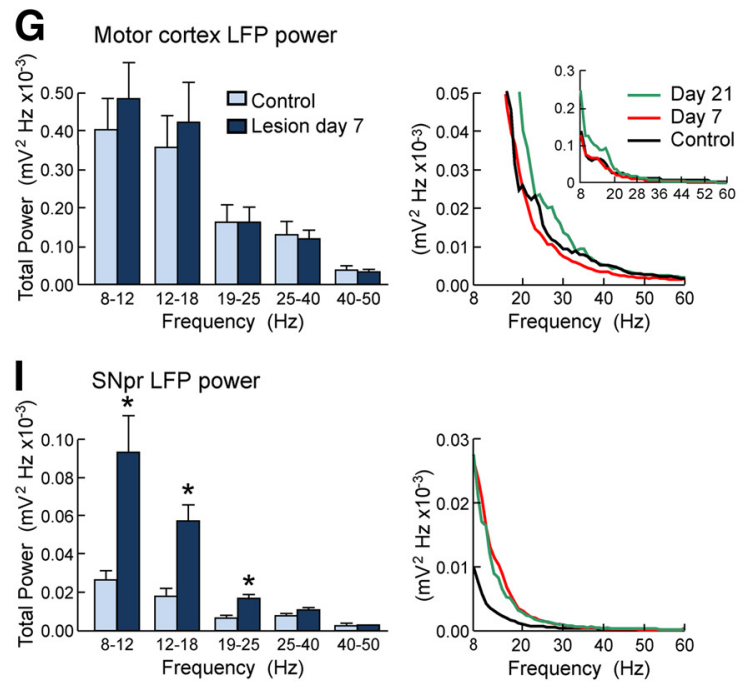

K Motor cortex-SNpr LFP coherence
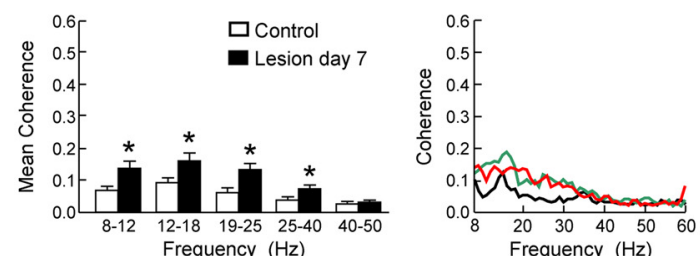

B

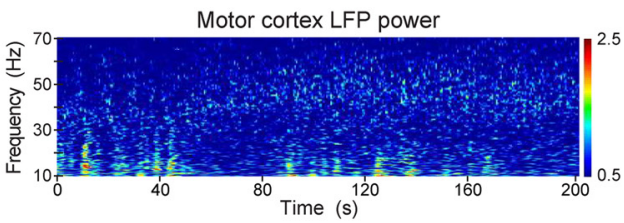

D

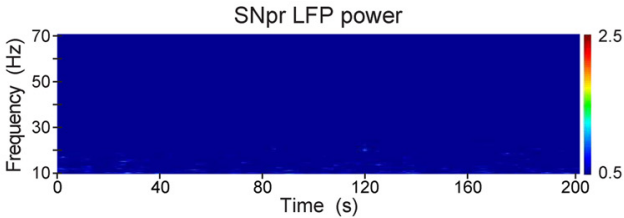

$\mathbf{F}$

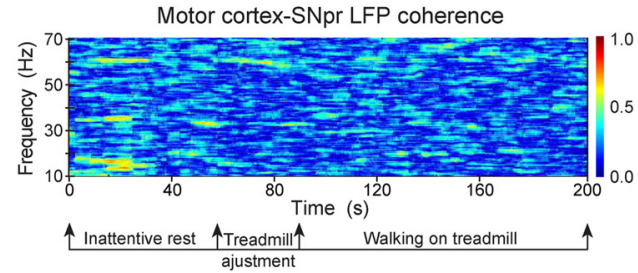

Treadmill Walking
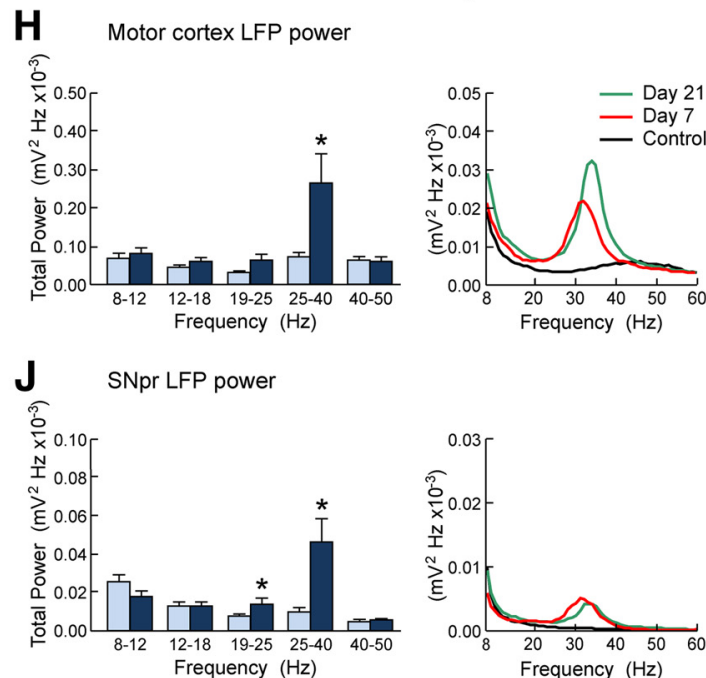

L Motor cortex-SNpr LFP coherence
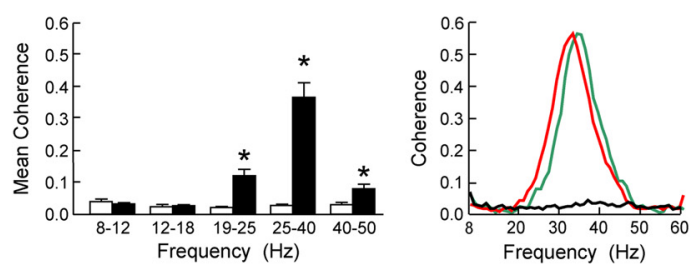

Figure 1. Power and coherence of motor cortex and SNpr LFP activity in the dopamine-cell-lesioned hemisphere and in intact rats during rest and treadmill walking. $\boldsymbol{A}-\boldsymbol{F}$, Representative wavelet-based scalograms represent the time-frequency plots of LFP spectral power in the motor cortex $(\boldsymbol{A}, \boldsymbol{B})$ and $S \mathrm{Npr}(\boldsymbol{C}, \boldsymbol{D})$ during inattentive rest, adjustment as the treadmill is started, and treadmill walking. Spectral power was plotted on a logarithmic scale with greater power being represented by red colors. FFT-based spectrograms ( $\boldsymbol{E}, \boldsymbol{F})$ show coherence between motor cortex and SNpr over the same time interval. Note the emergence of high beta/low gamma $(30-35 \mathrm{~Hz})$ frequency oscillations and increased coherence between the motor cortex and SNpr LFP activity at the transition from rest to walk in the dopamine-cell-lesioned hemisphere. A modest, more diffused band is present in the $40-50 \mathrm{~Hz}$ range in the motor cortex of the control rats during treadmill walking. $\mathbf{G}-\boldsymbol{L}$, Bar graphs represent mean total LFP power in the motor cortex $(\boldsymbol{G}, \boldsymbol{H})$ and SNpr $(\boldsymbol{I}, \boldsymbol{J})$ and motor cortex-SNpr coherence $(\boldsymbol{K}, \boldsymbol{L})$ within a series of frequency ranges: alpha $(8-12 \mathrm{~Hz})$, low beta $(12-18 \mathrm{~Hz}$ and $19-25 \mathrm{~Hz})$, high beta/low gamma $(25-40 \mathrm{~Hz})$, and gamma $(40-50 \mathrm{~Hz})$ in intact rats $(N=8)$ and day 7 postlesion in dopamine-cell-lesioned hemispheres $(N=10)$. Linear graphs show averaged LFP power spectra and motor cortex-SNpr coherence from 8 to $60 \mathrm{~Hz}$ for the same rest and walking epochs from the intact hemisphere (black) and from lesioned hemispheres at day 7 postlesion (red), and for day 21 postlesion (green, $N=8$ ). The insert in $\mathbf{G}$ shows the same averaged cortical LFP power spectra with an expanded $y$-axis. During inattentive rest, SNpr power and motor cortex-SNpr coherence are increased in frequencies between 8 and $25 \mathrm{~Hz}$ and 8 and $40 \mathrm{~Hz}$, respectively, while walking epochs are characterized by increased coherence and power in both motor cortex and SNpr specifically in the $25-40 \mathrm{~Hz}$ frequency range. *Significant difference between intact and lesion hemispheres. 


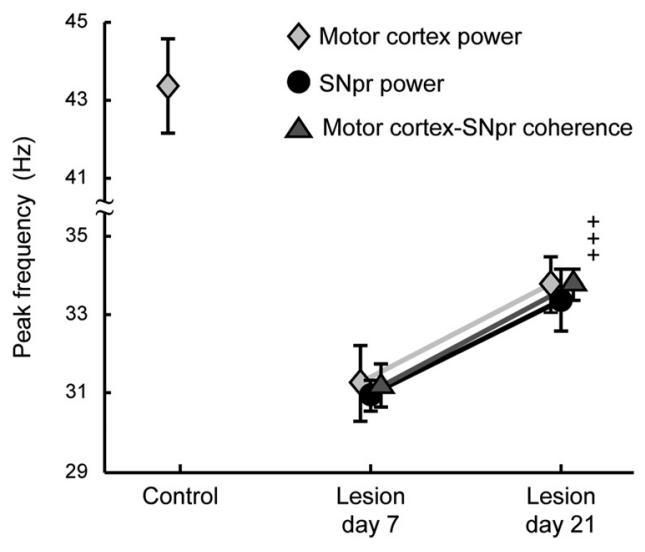

Figure 2. Mean frequency of the significant peaks in motor cortex and SNpr power and in motor cortex-SNpr coherence in dopamine-lesioned rats at day 7 and day 21 postlesion during walking. The scatter plot also shows the mean frequency peak of LFP power in intact motor cortex (no peak was observed in the SNpr). Only the set of lesioned animals with data from both day 7 and day 21 were considered $(N=8)$. Note that the mean frequency of significant peaks in motor cortex power and motor cortex-SNpr coherence in the $30-35 \mathrm{~Hz}$ range during walking is significantly increased between the 7 th and 21 st days postlesion. ${ }^{+}$Significant difference from day 7 (two-way RM ANOVA, $p<0.05$ ).

walking on the treadmill as long as they were oriented in the direction ipsiversive to the lesioned hemisphere. In contrast to the changes observed during epochs of inattentive rest, SNpr LFP power increases were focused in the $25-40 \mathrm{~Hz}$ high beta/low gamma frequency range during ipsiversive treadmill walking. As is evident in Figure 1, $C, D$, and $J$, increases in LFP power in the 25-40 Hz range were associated with a clear peak in the LFP power spectra with a mean frequency of $30.9 \pm 0.4 \mathrm{~Hz}(N=8)$ at day 7, which was significantly increased to $33.4 \pm 0.8 \mathrm{~Hz}(N=8)$ at day 21 (two-way RM ANOVA, $p<0.05$; Fig. 2). During walking epochs, total SNpr LFP power over the 25-40 Hz range was approximately five times greater in the lesioned hemisphere of the hemiparkinsonian rat than in the control rat.

\section{Oscillatory LFP activity in motor cortex after dopamine cell lesion}

To determine whether the patterns of change in LFP power observed in the SNpr after loss of dopamine were associated with comparable changes in LFP activity in the motor cortex, we next analyzed LFPs recorded from motor cortex layer 5/6 in the same rats (Fig. $1 A, B, G, H)$. Overall, LFP power in the cortex was significantly greater than in the SNpr in both control and dopamine-depleted rats $(p<0.001)$, consistent with the more regular columnar alignment of neurons and their processes in the cortex. During rest epochs at day 7 postlesion, no significant increase was observed in cortical LFP power in the $8-25 \mathrm{~Hz}$ range, however, in contrast to the marked increases in LFP in the $8-25 \mathrm{~Hz}$ range observed in the SNpr. There were, nevertheless, significant increases in LFP power in the $8-12$ and $12-18 \mathrm{~Hz}$ ranges by day 21 (Fig. $1 G$ ).

A very different picture emerged in the motor cortex during treadmill walking after dopamine cell lesion; under these conditions, changes observed in the SNpr were associated with dramatic changes in cortical LFP power. Control rats showed a modest but significant spectral peak with a mean frequency of $43.4 \pm 1.2 \mathrm{~Hz}(N=8)$ in LFPs recorded from the motor cortex (Fig. $1 B, H$ ). After dopamine cell lesion, this modest peak in the $40-45 \mathrm{~Hz}$ range was no longer apparent, and a dramatic increase in motor cortex LFP power emerged in the $25-40 \mathrm{~Hz}$ range, evident at day 7 and day 21 after dopamine cell lesion during treadmill walking (Fig. $1 A, B, H$ ). At day 7 , the increased power was focused around a spectral peak with a mean frequency of $31.3 \pm 1.0 \mathrm{~Hz}(N=8)$. At day 21 , the peak was significantly higher, with a mean frequency of $33.8 \pm 0.7 \mathrm{~Hz}(N=8$, two-way RM ANOVA, $p<0.05$; Fig. 2), consistent with the trend toward a higher mean frequency observed in the SNpr.

\section{Motor cortex-SNpr coherence after dopamine cell lesion}

To gain further insight into relationships between oscillatory LFP activity in the motor cortex and SNpr after dopamine cell lesion, coherence between motor cortex and SNpr LFPs was assessed during rest and walk epochs. Results showed that increases in SNpr LFP power were consistently associated with increases in motor cortex-SNpr coherence during both inattentive rest and treadmill walking after dopamine cell lesion. However, increases in coherence were considerably greater during the walking epochs, as shown in the representative wavelet-based spectrogram (Fig. $1 E, F$ ) and averaged spectra of FFT-based coherence (Fig. $1 K, L)$. During inattentive rest epochs, motor cortex-SNpr LFP coherence was significantly but modestly increased throughout the $8-40 \mathrm{~Hz}$ range, with values in the lesioned rats approximately twice those of the controls. During treadmill walking, however, motor cortex-SNpr coherence was significantly and robustly increased in the high beta/low gamma range. The mean peak frequencies of the increases in motor cortex-SNpr coherence during walking epochs were $31.2 \pm 0.5 \mathrm{~Hz}$ at day 7 and $33.0 \pm 0.4$ at day $21(N=8)$. These were well aligned with the mean peak frequencies in spectral power in motor cortex and SNpr and significantly increased over time (two-way RM ANOVA, $p<0.05$; Fig. 2). Mean motor cortex-SNpr coherence in the $30-36 \mathrm{~Hz}$ range at day 7 was $0.46 \pm 0.05(N=9)$ in the dopamine-depleted hemisphere, 15 times greater than in the intact rat over the same range $(0.03 \pm 0.002, N=8)$.

\section{Effect of L-dopa treatment on LFP power and motor cortex-SNpr coherence}

To confirm that loss of dopamine was associated with the appearance of coherent high beta/low gamma oscillatory activity in motor cortex and SNpr, L-dopa was administered 21-24 d after lesion and the effects on motor cortex and SNpr LFP power and coherence were measured during treadmill walking.

As previously reported (Brazhnik et al., 2009; Avila et al., 2010) L-dopa ( $5 \mathrm{mg} / \mathrm{kg}$, i.p.) administration was associated with a dramatic reduction in SNpr LFP power in the 25-40 Hz range in the dopamine-depleted hemisphere during walking epochs, and the recovery of the rat's ability to walk in both ipsiversively and contraversively, relative to the unilateral lesion, on the circular treadmill. The present results show that the L-dopa-induced reduction in SNpr power is associated with a comparable decrease in motor cortex LFP power and motor cortex-SNpr coherence in the $25-40 \mathrm{~Hz}$ range (Fig. 3). Interestingly, although LFP power was dramatically reduced after the $5 \mathrm{mg} / \mathrm{kg}$ dose of L-dopa, modest peaks were still evident in the cortical power spectra in the $25-50 \mathrm{~Hz}$ range and in motor cortex-SNpr coherence. However, these peaks in power and coherence spectra were significantly shifted to higher frequencies following L-dopa (for cortical power: from $33.3 \pm 0.4$ to $39.2 \pm 0.9 \mathrm{~Hz}, N=8$; for coherence: from $33.5 \pm 0.5$ to $38.8 \pm 0.3, p<0.001, N=8$ ), closer to those observed in control rats during treadmill walking. As shown in Figure 3, subsequent administration of the D2 antagonist eticlopride $(0.2 \mathrm{mg} / \mathrm{kg}$, i.p.) reversed the effect of L-dopa on LFP power in high beta frequency ranges and restored motor cortex-SNpr 
coherence. The effect of L-dopa on power during inattentive rest was not quantified in the present study, as periods of inattentive rest were relatively infrequent after L-dopa administration.

These results confirm the critical role of tonic loss of dopamine receptor stimulation and, in particular, D2 receptor stimulation, in mediating the increases in LFP and coherence in motor cortex and SNpr associated with injections of 6-OHDA into the medial forebrain bundle.

\section{Directionality and timing relationships between LFP oscillations in motor cortex and SNpr}

The presence of highly coherent bands of $30-35 \mathrm{~Hz}$ oscillatory activity in the LFP recorded from motor cortex and SNpr of the dopamine-depleted hemisphere during treadmill walking implies that the spiking activity of neuronal populations in both areas is relatively synchronized in this frequency range and phase-locked to these oscillations. Assuming the troughs of the LFP oscillations reflect the period of net peak depolarization of the neurons surrounding the recording electrode, it is reasonable to hypothesize, as a starting point, that the LFP troughs provide an indication of the time when these neurons are most likely to be spiking and are there-

fore useful for gaining insight into timing relationships between synchronized spiking in the two regions.

Two different approaches were taken to assess the directionality of information flow and time lags between the 25-40 Hz LFP oscillations in the motor cortex and the SNpr in the dopaminedepleted hemisphere during treadmill walking (Fig. 4). First, lag times between coherent LFP oscillations in motor cortex and SNpr during walking epochs were calculated as the shift at maximum cross-correlation between the two LFP signals filtered at 25-40 Hz. For this analysis, a positive lag indicates that the peak of the LFP oscillation in the cortex is occurring before the nearest peak of the LFP oscillation in the SNpr and a negative lag indicates the reverse. Overall, the data showed no clear directionality as to which area is leading in producing the $25-40 \mathrm{~Hz}$ oscillatory activity. In control rats $(N=8)$, LFP oscillations in motor cortex and SNpr were typically offset by less than $\pm 100 \mathrm{~ms}$ (mean lag \pm SD was $-1.17 \pm 110.39 \mathrm{~ms}$; Fig. $4 E, H)$. In contrast, in lesioned animals $(N=8)$, the filtered LFP activity from both regions showed considerably greater temporal coupling and much smaller phase shifts: LFPs were offset by less than \pm 5 ms (mean lag \pm SD: $0.34 \pm 4.28 \mathrm{~ms}$ at day 7 and $-0.08 \pm 10.33 \mathrm{~ms}$ at day 21 ; Fig. $4 F-H$ ). The SNpr preceded the cortex (negative lags) in $63 \%$ of the epochs in control rats, in $50 \%$ of the epochs in the dopamine-depleted rats at day 7 , and in $35 \%$ of the epochs at day 21 (Fig. 4C). Thus, while in control animals the LFPs in the SNpr exhibit a slight tendency to lead the LFPs recorded in the motor cortex, in lesioned animals the filtered LFP activity from both regions presented a high temporal coupling with much smaller phase shifts.
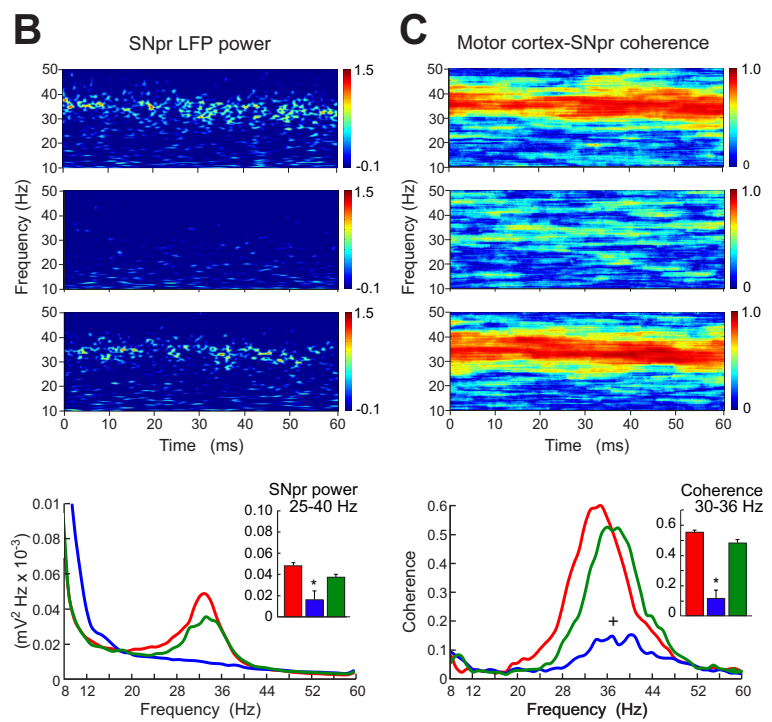

Figure 3. The effect of L-dopa treatment on LFP oscillatory activity in the motor cortex (MCX) and SNpr in the lesioned hemi(a) during walking. $\mathbf{A}-\boldsymbol{C}$, Linear plots of averaged power spectra and motor cortex-SNpr coherence and wavelet-based greatere frequencies of cortical power and coherence spectral peaks during L-dopa treatment are significantly higher than frequencies of spectral peaks for control and posteticlopride walking at day 21 (one-way RM ANOVA, $p<0.001$ ).

Second, analysis of the relationships between the fluctuations in LFP amplitude, i.e., the envelopes of the motor cortex and SNpr 25-40 Hz LFP oscillations, was performed. The envelopes of amplitude fluctuations of both cortical and SNpr $25-40 \mathrm{~Hz}$ LFP oscillations showed power (and coherence) in a broad range of frequencies $<10 \mathrm{~Hz}$, with no evidence of a significant peak emerging in the distribution of power within that range (data not shown). Further examination of the lag and directionality of the envelope of LFP amplitude fluctuations in the two brain regions was consistent, although in a wider temporal scale, with analysis of lag and directionality of the 25-40 Hz LFP rhythms themselves: the shifts in the peaks of cross-correlations of the SNpr and cortex LFP envelopes were larger and more divergent in control than in lesioned animals and, in all cases, no marked directionality was found (Fig. $4 D, I-L)$. Data showed mean lag times ( \pm SD) of $-378 \pm 10531 \mathrm{~ms}$ in controls and $-138 \pm 2432 \mathrm{~ms}$ at day 7 and $-95 \pm 3706 \mathrm{~ms}$ at day 21 postlesion. On average, SNpr was shown to be leading in 55\% of the epochs in the control rats and in $55 \%$ in lesioned animals at day 21 , while cortex was leading in $53 \%$ at day 7 (Fig. 4D).

Collectively, these results show a strong effect of loss of dopamine on lag times between LFP oscillations in the $25-40 \mathrm{~Hz}$ range. However, the very short (near zero) lag times and lack of consistent directionality of information flow evident in temporal relationships between LFPs from the motor cortex and SNpr from walking epochs in the dopamine-depleted hemisphere raised questions about the phase relationships between the LFP oscillations and spiking activity in these regions.

Anatomical considerations of connections between the motor cortex and basal ganglia have historically led to a focus on the 

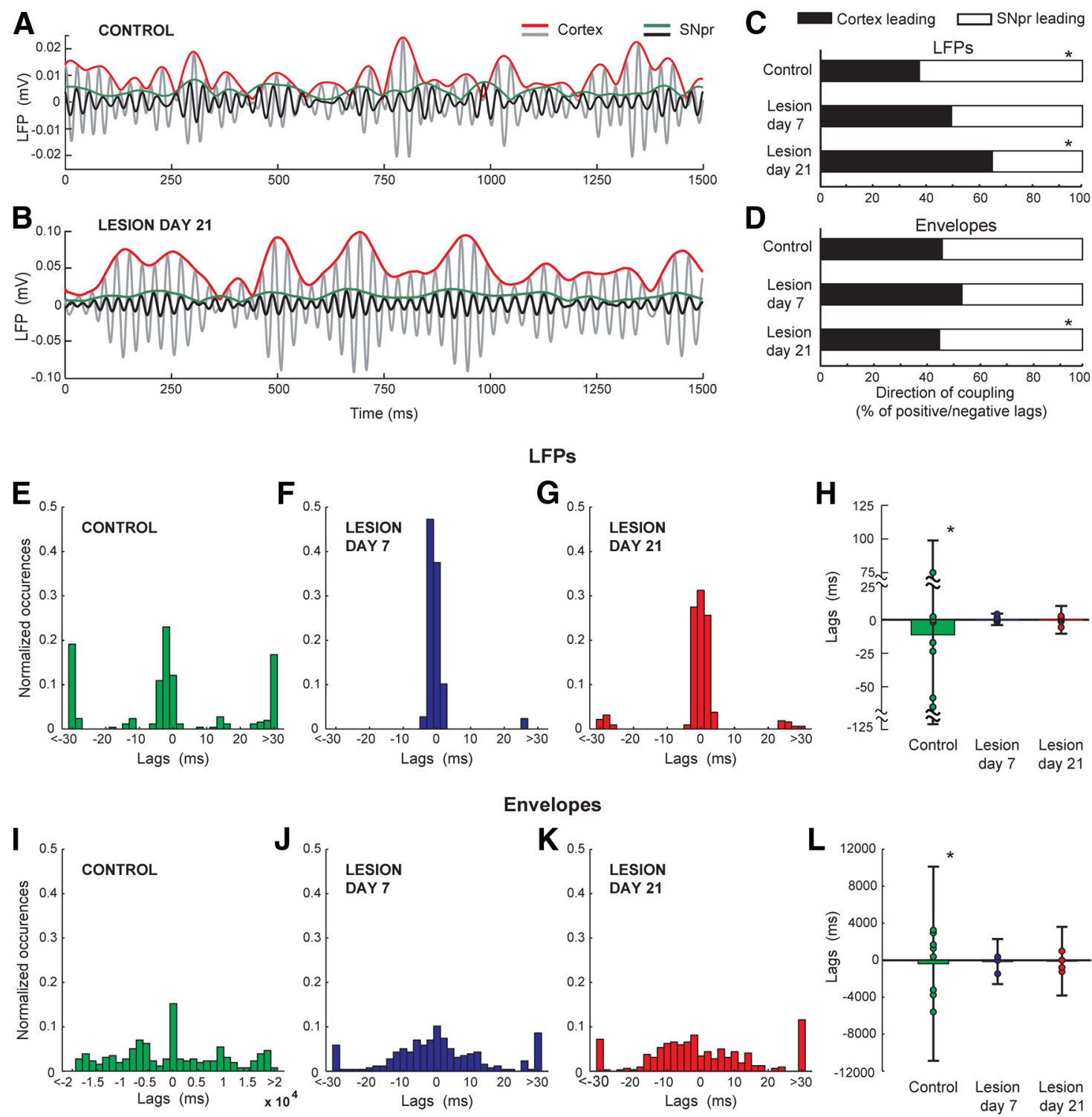

Figure 4. Characteristics of the high beta/low gamma frequency coupling of LFP oscillations in the motor cortex and SNpr of control and 6-OHDA-lesioned animals during walk. $\boldsymbol{A}, \boldsymbol{B}$, Simultaneous LFP recordings from motor cortex and SNpr filtered at $25-40 \mathrm{~Hz}$ and overlaid with an outline (red, green) representing the envelope of the power amplitude fluctuations calculated by the Hilbert transform in a control animal $(\boldsymbol{A})$ and in an animal day 21 postlesion $(\boldsymbol{B})$. C, $\boldsymbol{D}$, Estimated direction of coupling between cortex and SNpr for control $(N=8)$ and lesioned animals on day $7(N=8)$ and day $21(N=8)$; cortex is leading SNpr when cortex-SNpr lags are positive, and SNpr is leading cortex when lags are negative. The lags in $C$ were calculated as the shift at maximum cross-correlation between the filtered LFP signals and in $\boldsymbol{D}$ as the shift at maximum cross-correlation between LFP envelopes. $\boldsymbol{C}, \boldsymbol{D}$, Bars show the proportion of positive versus negative lags. $\boldsymbol{E}-\boldsymbol{G}$, Histograms of the distributions of the motor cortex-SNpr lag durations estimated by LFP cross-correlations for control animals $(\boldsymbol{E})$ and lesioned animals at day $7(\boldsymbol{F})$ and day $21(\boldsymbol{G})$ postlesion. $\boldsymbol{H}$, Means and standard deviations of the lag times in $\boldsymbol{E}-\boldsymbol{G}$, where the circles represent the mean lags of individual animals. $\boldsymbol{I} \boldsymbol{K} \boldsymbol{K}$, Histograms of the distributions of the motor cortex-SNpr lag durations estimated from the cross-correlations of the envelopes for the same three conditions. Note the different scales in $\boldsymbol{I}$ compared with $\boldsymbol{J}$ and $\boldsymbol{K}$. $\boldsymbol{L}$, Means and standard deviations of the lag times in $\boldsymbol{I} \boldsymbol{- K}$. Note the near-zero mean lag in the lesion animals $(0.34 \mathrm{~ms}$ at day 7 and -0.08 at day 21$)$ between cortical and SNpr LFPs $(\boldsymbol{F}-\boldsymbol{H})$. Additionally, the mean lags of the LFP envelopes were significantly lower in the lesioned animals ( $-138.17 \mathrm{~ms}$ at day 7 and $-95.37 \mathrm{~ms}$ at day 21$)$ than in controls $(-378.46 \mathrm{~ms}) .{ }^{*}$ Statistical significance $(p<0.05)$.

flow of information from cortex to the striatum and from the striatum to the SNpr via the direct and indirect pathways. The latter involves synapses in the GPe and the STN. Another route, the hyperdirect pathway, involves projections from the cortex to the STN and from the STN to the SNpr, and would be expected to produce a shorter lag time between cortical output and $\mathrm{SNpr}$ activation (Albin et al., 1989; Alexander and Crutcher, 1990; DeLong, 1990; Nambu, 2004).

If motor cortex-SNpr LFP phase relationships are predictive of the timing of spikes in the motor cortex relative to the SNpr in the dopamine-depleted hemisphere during treadmill walking, the near-zero lag data are indicating that spiking activity in the two brain regions is, on average, relatively synchronous. This scenario, however, is inconsistent with predictions of information flow from motor cortex to SNpr and anatomical considerations of corticobasal ganglia connectivity. To investigate this issue, we next examined the phase relationships between spiking activity and LFPs in the two brain regions during epochs of peak coherence and power.

\section{Spike-LFP relationships in motor cortex and SNpr during walking}

Several steps were taken to further examine the extent to which spiking activity in motor cortex and SNpr was phase-locked to 
simultaneously recorded LFP activity in the motor cortex and SNpr in the 25-40 $\mathrm{Hz}$ range, and to assess the timing relationships between synchronized spiking in the two brain regions. First, well sorted cortical neurons with firing rates $\geq 2 \mathrm{~Hz}$ from both dopamine-depleted and control animals were classified as putative pyramidal neurons or putative interneurons according to the duration of their waveform using $k$-means $(k=2)$ cluster analysis (Fig. 5A). Both groups of cells fit a normal distribution, with estimates of the mean duration of $0.52 \pm 0.008 \mathrm{~ms}(N=$ 90) for pyramidal neurons and $0.20 \pm$ $0.011 \mathrm{~ms}(N=44)$ for interneurons. A significance test (two-way ANOVA) on the different populations showed that the distributions of waveform durations between interneurons and pyramidal neurons were statistically different for both control and lesioned groups $(p<0.01)$; waveform durations of pyramidal neurons from control and lesioned rats were not significantly different from one another $(p=0.34)$, nor were waveform durations of interneurons from the control and lesioned populations ( $p=0.07$ ). Firing rates of pyramidal neurons were typically lower than $25 \mathrm{~Hz}$. Figure $5 A$ shows the distribution of firing rates of neurons with rates $\geq 2 \mathrm{~Hz}$. If neurons with rates $\geq 0.02 \mathrm{~Hz}$ were included in the dataset, pyramidal neurons with spontaneous activity during walking had a mean frequency of $6.6 \pm 1.1 \mathrm{~Hz}, N=40$ for control and $9.1 \pm 1.0 \mathrm{~Hz}, N=94$ for lesioned animals, while interneuron rates were distributed over a wider range $(18 \pm 4.5 \mathrm{~Hz}$, $N=19$ for control and $27.0 \pm 3.7 \mathrm{~Hz}, N=$ 26 for lesioned). Firing rates of SNpr neurons in control rats were $24.3 \pm 2.0(N=$ 61); rates were significantly increased in the dopamine-depleted hemisphere (33.4 $\pm 2.2, N=76$; Mann-Whitney rank sum test, $p=0.006$ ).

Second, the peak-to-trough values around time 0 of the spike-triggered LFP waveform averages were used to evaluate the extent of phase-locking of the spike train to simultaneously recorded LFP. STWA peak-to-trough amplitude averages were generated from pyramidal and interneuron neuronal spike trains referenced to corresponding periods of motor cortex LFP filtered at either $12-18 \mathrm{~Hz}$ or $25-40 \mathrm{~Hz}$ (Fig. 5B). Similarly, STWAs were generated from simultaneously recorded SNpr spike trains referenced to SNpr LFPs in the same frequency ranges. Interspike intervals from the same epochs were shuffled 20 times and after each shuffle, an additional STWA was generated. The peak-to-trough amplitude of the unshuffled STWA was considered to reflect significant phase-locking of the spikes to the LFP if it exceeded that of the mean STWA amplitude for the
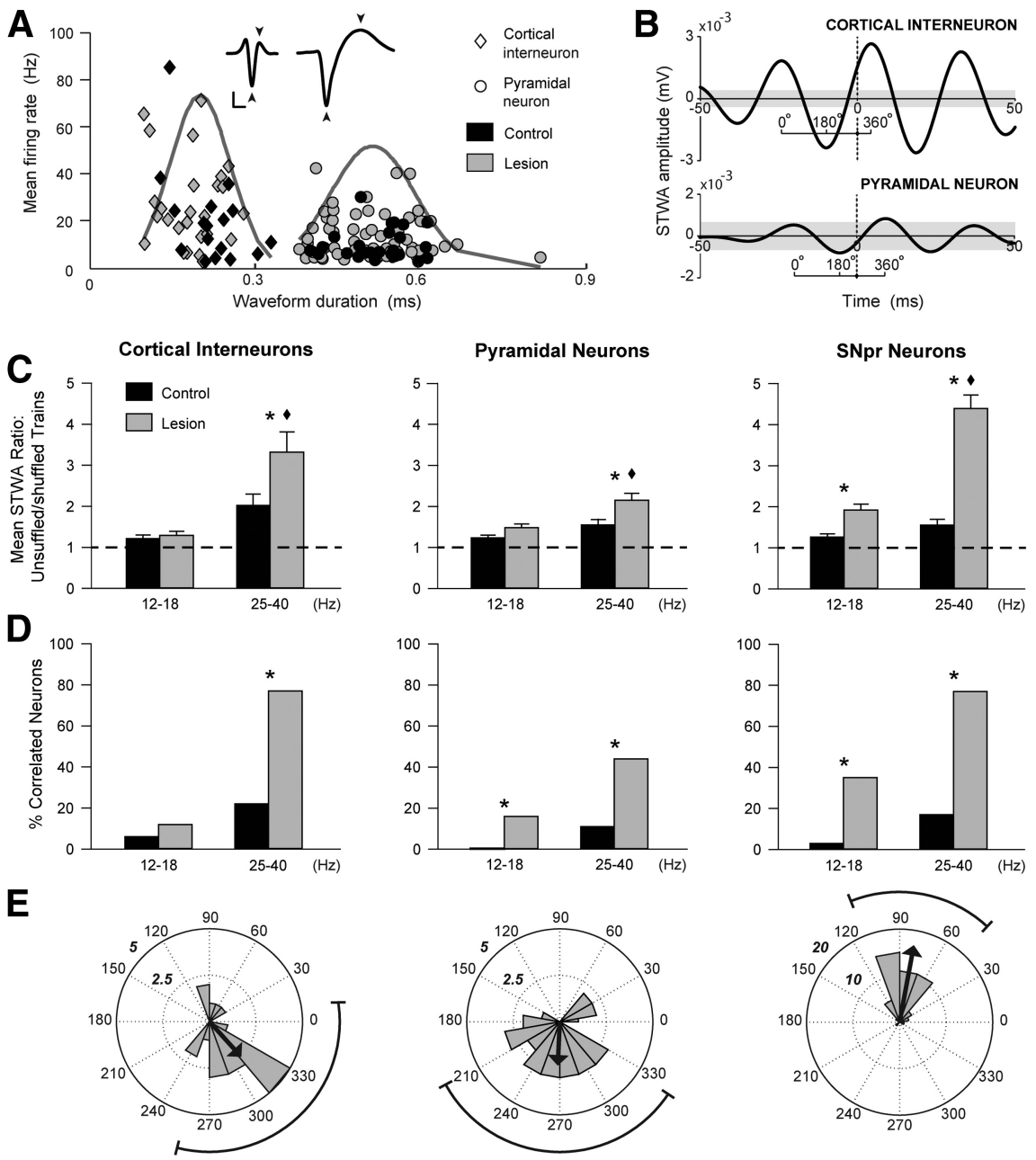

Figure 5. Analysis of spike-LFP relationships in motor cortex and SNpr during treadmill walking. $\boldsymbol{A}$, Scatter plot shows the distribution of waveform duration (trough to peak; arrowheads; scale: $0.2 \mathrm{mV}, 0.2 \mathrm{~ms}$ ) and firing rate of layer $5 / 6$ cortical neurons recorded from day 7 dopamine-lesioned and control rats for neurons with firing rates $\geq 2 \mathrm{~Hz}$. Cortical neurons were clustered into two groups based on the distributions of waveform duration ( $k$-means, $k=2)$, which were classified as interneurons $(N=43)$ or pyramidal neurons $(N=90)$. Interneurons and pyramidal neurons waveform distributions were shown to be statistically different both for control and lesioned (two-way ANOVA, $p<0.01$ ). Gray curves illustrate the normal characteristics of the distributions of waveform durations for each cell type (scaled with a factor of 10 for purposes of visualization). $\boldsymbol{B}$, Representative examples of STWA for cortical interneurons (top) and pyramidal spike trains (bottom) paired with cortical LFPs bandpass filtered at $25-40 \mathrm{~Hz}$, with phase-lock values of $311^{\circ}$ and $245^{\circ}$, respectively. The shaded area indicates the range of the mean peak-to-trough amplitude \pm 3 SD of 20 STWAs - each calculated by pairing the same LFP with a shuffled version of the spike train. When the original unshuffled STWAs peak-to-trough values were outside of this range, spikes were considered significantly phase-locked to LFP. C, Bar graphs show mean ratios between peak to trough amplitudes of the original STWA and the mean of 20 shuffled STWAs for LFPs in the frequency ranges $12-18$ and $25-40 \mathrm{~Hz}$ for putative cortical pyramidal and interneuronal neurons and SNpr neurons. Dashed line denotes ratio of 1 . *Significant difference between control and lesion within a frequency range $(p<0.05)$; Statistical significance across frequency ranges within lesioned or controls $(p<0.05)$. D, Bar graphs show the proportion of spike trains significantly phase-locked to locally recorded LFPs in the two frequency ranges. *Proportions of phase-locked spike trains are significantly greater in the dopamine-lesioned hemispheres compared with control for both frequency ranges $\left(\chi^{2}, p<0.05\right)$. $\boldsymbol{E}$, Phase plots show the distributions of phase relationships between spikes and cortical LFPs for those spike trains showing significant phase-locking to local LFP in D. Spikes were significantly oriented to high beta/low gamma LFP cortical oscillations for interneurons, pyramidal neurons, and SNpr neurons (Rayleigh, $p<0.005$ ). Arrows reflect a measure of the strength of concentration of the distribution of the mean phase values from spike trains in $D$, normalized to the radius of the circular plot.

shuffled data plus three times the standard deviation of the shuffled data.

Figure $5 C$ shows a comparison of the mean ratios of unshuffled to shuffled STWA peak-to-trough amplitudes of the three types of neurons referenced to LFPs filtered in two frequency ranges. The unshuffled/shuffled STWA amplitude ratios from spike trains recorded in the lesioned animals were significantly greater than the unshuffled/shuffled STWA amplitude ra- 
tios in controls in the $25-40 \mathrm{~Hz}$ frequency range during treadmill walking for interneurons, pyramidal neurons, and SNpr neurons. For SNpr neurons, ratios were also increased in the lesioned hemisphere in the $12-18 \mathrm{~Hz}$ frequency range (two-way ANOVA, $p<0.05$ ). These data show that the neuronal spiking of all three types of neurons is more phase-locked to the $25-40 \mathrm{~Hz}$ rhythms in the dopamine-depleted animals than in the control animals. Interestingly, the ratio of unshuffled to shuffled STWAs in the lesioned hemisphere in the $25-40 \mathrm{~Hz}$ range was lower for the pyramidal neurons than for the other two populations. It shows that the pyramidal neurons, as a population, are less phase-locked to the $25-40 \mathrm{~Hz}$ oscillatory rhythm than the other two neuronal populations (Kruskal-Wallis, one-way ANOVA, $p<0.001)$.

Figure $5 D$ shows a second way of looking at the phase-locking of these three populations to the high beta/low gamma rhythm: the relative proportion of neurons showing a significant correlation between spiking activity and LFP phase over the $25-40 \mathrm{~Hz}$ frequency range during treadmill walking. The original spike trains were considered significantly phase-locked to the LFP if their STWA peak-to-trough amplitudes exceeded the value of the mean plus 3 SDs of the STWAs from the spike train versions where spike timing was randomized. The percentage of neurons that were significantly phase-locked was greater in the lesioned animals (44\% for pyramidal neurons, $77 \%$ for interneurons, and $77 \%$ for SNpr neurons) than in the controls (11\% for pyramidal neurons, $22 \%$ for interneurons, and $17 \%$ for SNpr neurons). Collectively, these data show that in the lesioned animal, there is a significant increase in the proportion of neurons in motor cortex and SNpr that become synchronized with the dominate LFP oscillations occurring in the $25-40 \mathrm{~Hz}$ range during treadmill walking. As with the mean ratio data shown in Figure $5 C$, the proportion of phase-locked neurons within these brain regions is lowest for the cortical pyramidal neurons.

For further analysis of relationships between synchronized spiking and LFP oscillations, the phase relationships between spiking and LFP oscillations were calculated for those spike trains where spikes were significantly correlated with the LFPs (Fig. $5 D$ ). The results (Fig. $5 E$ ) show that the expectation that spiking is more likely to occur during the descending phase and near the valley of the LFP oscillations (as discussed in the previous section) was not valid for the cortical neuronal populations recorded in the present study. Both putative pyramidal neurons and interneurons fired more frequently during the ascending phase of the LFP oscillation. In all three populations, spiking was significantly phase oriented; that is, it tended to occur around a particular phase of the oscillation (Rayleigh, $p<0.005$ ). Moreover, the cortical neurons presented a distribution of spike-LFP phases significantly different from that of the neurons in the SNpr (Mardia-Watson-Wheeler, $p<0.001)$. Spikes of SNpr neurons tended to be phase-locked to the cortical LFPs with a mean angle \pm angular deviation of $79 \pm 35^{\circ}$, while the spikes of pyramidal neurons were phase-locked with a mean angle of $261 \pm 54^{\circ}$ and the cortical interneurons a mean angle of $312 \pm 58^{\circ}$. On average, for LFP cortical oscillations filtered at $25-40 \mathrm{~Hz}$ with a mean frequency of $31 \mathrm{~Hz}$, SNpr neurons were firing 17 ms after cortical pyramidal neurons. The phase distributions of the pyramidal neurons and the interneurons were not significantly different (Mardia-Watson-Wheeler, $p=0.135$ ). Thus, although cortical and SNpr LPFs tended to show near-zero lag (Fig. 4), the average offset in time of spiking of cortical neurons and SNpr neurons with significant phase-locking to the LFPs was approximately half the period of the main oscillation. These results are consistent with cortical activity entraining basal ganglia output via a multisynaptic pathway.

\section{Discussion}

The present study examines the hypothesis that synchronized activity in basal ganglia output in Parkinson's disease reflects an enhanced tendency for basal ganglia activity to become entrained by cortical rhythms after loss of dopamine. Supporting this view are the current observations showing that over a range of frequencies and in two behavioral states, increases in oscillatory activity in the SNpr of the hemiparkinsonian rat are consistently accompanied by increases in coherence between motor cortex and SNpr LFPs. These observations are consistent with evidence that dopamine cell loss facilitates striatal transmission of corticostriatal input to downstream sites (Tseng et al., 2001; Murer et al., 2002; Mallet et al., 2006; Walters et al., 2007; Aravamuthan et al., 2008). Other results of this study, however, suggest that additional processes may be involved in determining the profile of activity in basal ganglia output after dopamine cell lesion. The most notable finding was the L-dopa-reversible, frequencyspecific tuning of the corticonigral network in the $30-35 \mathrm{~Hz}$ range in dopamine-cell-lesioned rats during treadmill walking. As there was no comparable peak in this frequency in the cortex of control animals, it was not evident why this oscillation becomes so prominent in basal ganglia output and in the corticonigral network during on-going motor activity after dopamine cell loss. Further perspective on this issue came from spike-LFP relationships of substantia nigra and motor cortex layer 5/6 neurons during treadmill walking. Although the LFPs in the motor cortex are closely aligned with those in the SNpr, the timing of the phase-locked spiking activity in the two regions is significantly offset. The latter observation is consistent with the hypothesis that cortical rhythms entrain basal ganglia activity more readily after loss of dopamine and leave open the possibility that feedback via thalamocortical projections contributes to the $30-35 \mathrm{~Hz}$ peak in the motor cortex.

The dramatically different patterns of power and coherence that were observed in the present study during treadmill walking versus inattentive rest highlight the importance of behavioral state when exploring mechanisms underlying the effect of dopamine loss on synchronized activity in the motor cortex and basal ganglia. The proposal that loss of dopamine promotes synchronization of basal ganglia activity by enhancing entrainment to cortical rhythms (Tseng et al., 2001; Murer et al., 2002) evolved from studies in anesthetized rats where a $\sim 1 \mathrm{~Hz}$ rhythm is widely expressed in the cortex (Steriade et al., 1993). The present study shows that the tendency for basal ganglia output to become coherent with cortical activity in the absence of significant change in spectral power in the cortex after loss of dopamine is not unique to the $\sim 1 \mathrm{~Hz}$ frequency range nor to anesthetized rats. At day 7 postlesion during inattentive rest, increases in SNpr power and motor cortex-SNpr coherence occurred in the absence of significant increases in motor cortex power, consistent with the idea that the cortical rhythms are being more effectively transmitted to downstream sites after loss of dopamine.

The extent to which alterations in striatal processing of cortical input underlie the synchronization of basal ganglia activity in higher frequency ranges after dopamine cell loss is an open question. Changes in striatal cholinergic function induced by loss of dopamine can promote the appearance of low beta range oscillations within the striatum (McCarthy et al., 2011), while interactions between STN and GPe appear to facilitate emergence of 
$\sim 20 \mathrm{~Hz}$ range activity in these nuclei in anesthetized rats during states of global excitation after loss of dopamine (Mallet et al., 2008a; Holgado et al., 2010). However, it is unclear what role these mechanisms play in generating increases in corticonigral coherence over the wider range of frequencies at which basal ganglia output becomes entrained under different behavioral states. In particular, it would be of considerable interest to understand why the $30-35 \mathrm{~Hz}$ oscillations are so prominent in the corticonigral network during walking in the hemiparkinsonian rat. No peak was evident in this frequency range in control rats, although these rats did have a modest LFP peak in the $40-45 \mathrm{~Hz}$ range in the motor cortex during walking, which was not associated with a coherent peak in SNpr LFP power. One possibility is that $30-35 \mathrm{~Hz}$ is a frequency range compatible with resonant activity in the dopamine-depleted basal ganglia-thalamocortical circuit in the rat. As high beta/low gamma range activity appears readily generated by activated cortical circuits (Murthy and Fetz, 1992; Fries et al., 2007; Tiesinga and Sejnowski, 2009; Kopell et al., 2010), this activity may emerge in the cortex of the lesioned hemisphere (Li et al., 2007; Dejean et al., 2009; Gradinaru et al., 2009) — perhaps as a compensatory response to motor symptoms or as a result of loss of cortical dopamine-and subsequently entrain the larger network, inducing recurrent activation at the appropriate phase. It should be noted that the present observations of increased motor cortex-SNpr coherence and high beta range power in active hemiparkinsonian rats are consistent with reports of coherence and increased oscillatory activity in motor cortex and STN in behaving rats with unilateral dopamine cell lesion (Sharott et al., 2005; Degos et al., 2009).

To gain further insight into motor cortex-SNpr relationships during periods of increased coherence and power during walking, temporal relationships between LFPs and spiking activity in the motor cortex and SNpr were explored. Data from LFP waveform cross-correlation analyses showed near zero lag times with the SNpr leading the cortex as often as the reverse in lesioned animals, inconsistent with the hypothesis that cortical rhythms are driving the synchronized activity in the SNpr. Variability in the effective direction of coupling between cortex and STN has also been observed using cortical magnetoencephalographic and STN LFP recordings in parkinsonian patients during periods when patients were off medication (Litvak et al., 2011). However, in the current study, in contrast to predictions from LFP lags, STWA analysis showed that spiking activity in the two regions was offset, synchronizing at different phases of the prominent $\sim 30 \mathrm{~ms}$ cortical oscillatory LFP cycle. While it is not possible to determine directionality from these data, the $\sim 17 \mathrm{~ms}$ offset in synchronized spiking in cortex and SNpr during walking in the hemiparkinsonian rat is consistent with cortical $30-35 \mathrm{~Hz}$ oscillations entraining basal ganglia activity via multisynaptic pathways and facilitating synchronization throughout the basal ganglia-thalamocortical loop (Brazhnik et al., 2011). Of interest in this regard are the small, but significant, increases in the peak frequency of LFP power in motor cortex and SNpr observed between days 7 and 21 postlesion, suggesting that ongoing synchronous activity promotes plasticity over time in the underlying network.

Forty-four percent of the cortical neurons identified as putative pyramidal neurons, based on waveform duration, were significantly phase-locked to the cortical $30-35 \mathrm{~Hz}$ LFP rhythm, compared with $77 \%$ of the putative interneurons and the $\mathrm{SNpr}$ neurons. While this indicates that only a subset of layer $5 / 6$ pyramidal neurons would appear to qualify for playing a role in entraining downstream activity after loss of dopamine, modeling studies have indicated that small populations of motor neurons can effectively transmit oscillatory signals downstream (Baker et al., 2003). In contrast, recent studies have shown that corticalspinal neurons in primates have faster conduction velocities and shorter duration action potentials than corticostriatal neurons, and are likely to be miscategorized as interneurons when using waveform duration to identify cell type (Pasquereau and Turner, 2011; Vigneswaran et al., 2011). While not yet confirmed for rodents (Mathai and Smith, 2011), the primate data emphasizes the limitations of categorizing cortical neurons by waveform duration.

In summary, results are consistent with a role for cortical input to basal ganglia in entraining synchronization in basal ganglia output in the awake hemiparkinsonian rat (Murer et al., 2002; Li et al., 2007; Gradinaru et al., 2009). The results also show that spectral power in motor cortex and basal ganglia output varies dramatically with behavioral state and, relative to inattentive rest, is notably enhanced during walking in the dopaminedepleted rat with motor deficits. In this context, it is tempting to view the $30-35 \mathrm{~Hz}$ oscillations in the dopamine-depleted rodent as the analog of the $15-30 \mathrm{~Hz}$ activity observed in the STN of parkinsonian patients (Priori et al., 2004; Gatev et al., 2006; Kühn et al., 2009; Zaidel et al., 2010) and hypothesize that the differences in peak frequencies in the two species are related to differences in the motor output required during recordings. However, recordings in patients during movements of the arm or hand (Jenkinson and Brown, 2011), studies in alert nonhuman dopamine-depleted primates where peak frequencies in basal ganglia synchronization are typically $<20 \mathrm{~Hz}$ (Priori et al., 2004; Heimer et al., 2006; Leblois et al., 2007; Goldberg and Bergman, 2011), and data from hemiparkinsonian rats during attentive rest (Brazhnik et al., 2009) collectively suggest that differences in motor behavior during alert states will not account for the variance in peak frequencies of basal ganglia oscillations across these populations. At issue is the functional significance of excessive synchronization of basal ganglia output, within any specific frequency range or within a range of frequencies. Hopefully, further exploration of the relationships between activity state, behavioral symptoms, and the processes causing excessive synchronization of basal ganglia activity across species will provide additional insight into fundamental mechanisms underlying the profound behavioral consequences of loss of dopamine.

\section{References}

Adhikari A, Topiwala MA, Gordon JA (2010) Synchronized activity between the ventral hippocampus and the medial prefrontal cortex during anxiety. Neuron 65:257-269.

Albin RL, Young AB, Penney JB (1989) The functional anatomy of basal ganglia disorders. Trends Neurosci 12:366-375.

Alexander GE, Crutcher MD (1990) Functional architecture of basal ganglia circuits: neural substrates of parallel processing. Trends Neurosci 13:266-271.

Alonso-Frech F, Zamarbide I, Alegre M, Rodríguez-Oroz MC, Guridi J, Manrique M, Valencia M, Artieda J, Obeso JA (2006) Slow oscillatory activity and levodopa-induced dyskinesias in Parkinson's disease. Brain 129:1748-1757.

Aravamuthan BR, Bergstrom DA, French RA, Taylor JJ, Parr-Brownlie LC, Walters JR (2008) Altered neuronal activity relationships between the pedunculopontine nucleus and motor cortex in a rodent model of Parkinson's disease. Exp Neurol 213:268-280.

Avila I, Parr-Brownlie LC, Brazhnik E, Castañeda E, Bergstrom DA, Walters JR (2010) Beta frequency synchronization in basal ganglia output during rest and walk in a hemiparkinsonian rat. Exp Neurol 221:307-319.

Baker SN, Pinches EM, Lemon RN (2003) Synchronization in monkey motor cortex during a precision grip task. II. Effect of oscillatory activity on corticospinal output. J Neurophysiol 89:1941-1953. 
Barthó P, Hirase H, Monconduit L, Zugaro M, Harris KD, Buzsáki G (2004) Characterization of neocortical principal cells and interneurons by network interactions and extracellular features. J Neurophysiol 92:600-608.

Baufreton J, Atherton JF, Surmeier DJ, Bevan MD (2005) Enhancement of excitatory synaptic integration by GABAergic inhibition in the subthalamic nucleus. J Neurosci 25:8505-8517.

Brazhnik E, Avila I, Novikov N, Bergstrom DA, Walters JR (2009) Functional correlates of exaggerated oscillatory activity in basal ganglia and impairment of motor activity in hemiparkinsonian rats. Soc Neurosci Abstr 35:567.24.

Brazhnik E, Hatch CE, Novikov N, McCoy AJ, Wahba MI, Cruz AV, Amirtharaj MJ, Ilieva NM, Walters JR (2011) Ventral medial thalamus contributes to increased high beta/low gamma coherence in the basal ganglia thalamocortical network in an awake behaving rodent model of Parkinson's disease. Soc Neurosci Abstr 37:811.23.

Brown P (2003) Oscillatory nature of human basal ganglia activity: relationship to the pathophysiology of Parkinson's disease. Mov Disord 18:357-363.

Brown P, Oliviero A, Mazzone P, Insola A, Tonali P, Di Lazzaro V (2001) Dopamine dependency of oscillations between subthalamic nucleus and pallidum in Parkinson's disease. J Neurosci 21:1033-1038.

Cruz AV, Mallet N, Magill PJ, Brown P, Averbeck BB (2011) Effects of dopamine depletion on information flow between the subthalamic nucleus and external globus pallidus. J Neurophysiol 106:2012-2023.

Degos B, Deniau JM, Chavez M, Maurice N (2009) Chronic but not acute dopaminergic transmission interruption promotes a progressive increase in cortical beta frequency synchronization: relationships to vigilance state and akinesia. Cereb Cortex 19:1616-1630.

Dejean C, Hyland B, Arbuthnott G (2009) Cortical effects of subthalamic stimulation correlate with behavioral recovery from dopamine antagonist induced akinesia. Cereb Cortex 19:1055-1063.

DeLong MR (1990) Primate models of movement-disorders of basal ganglia origin. Trends Neurosci 13:281-285.

Doyle LM, Kühn AA, Hariz M, Kupsch A, Schneider GH, Brown P (2005) Levodopa-induced modulation of subthalamic beta oscillations during self-paced movements in patients with Parkinson's disease. Eur J Neurosci 21:1403-1412.

Fogelson N, Williams D, Tijssen M, van Bruggen G, Speelman H, Brown P (2006) Different functional loops between cerebral cortex and the subthalmic area in Parkinson's disease. Cereb Cortex 16:64-75.

Fries P, Nikolić D, Singer W (2007) The gamma cycle. Trends Neurosci 30:309-316.

Gatev P, Darbin O, Wichmann T (2006) Oscillations in the basal ganglia under normal conditions and in movement disorders. Mov Disord 21:1566-1577.

Goldberg JA, Bergman H (2011) Computational physiology of the neural networks of the primate globus pallidus: function and dysfunction. Neuroscience 198:171-192.

Gradinaru V, Mogri M, Thompson KR, Henderson JM, Deisseroth K (2009) Optical deconstruction of parkinsonian neural circuitry. Science 324:354-359.

Heimer G, Rivlin-Etzion M, Bar-Gad I, Goldberg JA, Haber SN, Bergman H (2006) Dopamine replacement therapy does not restore the full spectrum of normal pallidal activity in the 1-methyl-4-phenyl-1,2,3,6tetra-hydropyridine primate model of Parkinsonism. J Neurosci 26:8101-8114.

Holgado AJ, Terry JR, Bogacz R (2010) Conditions for the generation of beta oscillations in the subthalamic nucleus-globus pallidus network. J Neurosci 30:12340-12352.

Isomura Y, Harukuni R, Takekawa T, Aizawa H, Fukai T (2009) Microcircuitry coordination of cortical motor information in self-initiation of voluntary movements. Nat Neurosci 12:1586-1593.

Jenkinson N, Brown P (2011) New insights into the relationship between dopamine, beta oscillations and motor function. Trends Neurosci 34:611-618.

Kopell N, Kramer MA, Malerba P, Whittington MA (2010) Are different rhythms good for different functions? Front Hum Neurosci 4:187.

Kühn AA, Tsui A, Aziz T, Ray N, Brücke C, Kupsch A, Schneider GH, Brown P (2009) Pathological synchronisation in the subthalamic nucleus of patients with Parkinson's disease relates to both bradykinesia and rigidity. Exp Neurol 215:380-387.

Leblois A, Meissner W, Bioulac B, Gross CE, Hansel D, Boraud T (2007)
Late emergence of synchronized oscillatory activity in the pallidum during progressive Parkinsonism. Eur J Neurosci 26:1701-1713.

Levy R, Hutchison WD, Lozano AM, Dostrovsky JO (2002) Synchronized neuronal discharge in the basal ganglia of parkinsonian patients is limited to oscillatory activity. J Neurosci 22:2855-2861.

Li S, Arbuthnott GW, Jutras MJ, Goldberg JA, Jaeger D (2007) Resonant antidromic cortical circuit activation as a consequence of high-frequency subthalamic deep-brain stimulation. J Neurophysiol 98:3525-3537.

Litvak V, Jha A, Eusebio A, Oostenveld R, Foltynie T, Limousin P, Zrinzo L, Hariz MI, Friston K, Brown P (2011) Resting oscillatory corticosubthalamic connectivity in patients with Parkinson's disease. Brain 134:359-374.

Mallet N, Ballion B, Le Moine C, Gonon F (2006) Cortical inputs and GABA interneurons imbalance projection neurons in the striatum of parkinsonian rats. J Neurosci 26:3875-3884.

Mallet N, Pogosyan A, Márton LF, Bolam JP, Brown P, Magill PJ (2008a) Parkinsonian beta oscillations in the external globus pallidus and their relationship with subthalamic nucleus activity. J Neurosci 28:1424514258.

Mallet N, Pogosyan A, Sharott A, Csicsvari J, Bolam JP, Brown P, Magill PJ (2008b) Disrupted dopamine transmission and the emergence of exaggerated beta oscillations in subthalamic nucleus and cerebral cortex. J Neurosci 28:4795-4806.

Mathai A, Smith Y (2011) The corticostriatal and corticosubthalamic pathways: two entries, one target. So what? Front Syst Neurosci 5:64.

McCarthy MM, Moore-Kochlacs C, Gu X, Boyden ES, Han X, Kopell N (2011) Striatal origin of the pathologic beta oscillations in Parkinson's disease. Proc Natl Acad Sci U S A 108:11620-11625.

Mitchell JF, Sundberg KA, Reynolds JH (2007) Differential attentiondependent response modulation across cell classes in macaque visual area V4. Neuron 55:131-141.

Murer MG, Tseng KY, Kasanetz F, Belluscio M, Riquelme LA (2002) Brain oscillations, medium spiny neurons, and dopamine. Cell Mol Neurobiol 22:611-632.

Murthy VN, Fetz EE (1992) Coherent 25- to 35-Hz oscillations in the sensorimotor cortex of awake behaving monkeys. Proc Natl Acad Sci U S A 89:5670-5674.

Nambu A (2004) A new dynamic model of the cortico-basal ganglia loop. Prog Brain Res 143:461-466.

Olsson M, Nikkhah G, Bentlage C, Björklund A (1995) Forelimb akinesia in the rat Parkinson model: differential effects of dopamine agonists and nigral transplants as assessed by a new stepping test. J Neurosci 15:3863-3875.

Parr-Brownlie LC, Hyland BI (2005) Bradykinesia induced by dopamine D2 receptor blockade is associated with reduced motor cortex activity in the rat. J Neurosci 25:5700-5709.

Pasquereau B, Turner RS (2011) Primary motor cortex of the parkinsonian monkey: differential effects on the spontaneous activity of pyramidal tract-type neurons. Cereb Cortex 21:1362-1378.

Priori A, Foffani G, Pesenti A, Tamma F, Bianchi AM, Pellegrini M, Locatelli M, Moxon KA, Villani RM (2004) Rhythm-specific pharmacological modulation of subthalamic activity in Parkinson's disease. Exp Neurol 189:369-379.

Sharott A, Magill PJ, Harnack D, Kupsch A, Meissner W, Brown P (2005) Dopamine depletion increases the power and coherence of betaoscillations in the cerebral cortex and subthalamic nucleus of the awake rat. Eur J Neurosci 21:1413-1422.

Shen KZ, Johnson SW (2005) Dopamine depletion alters responses to glutamate and GABA in the rat subthalamic nucleus. Neuroreport 16:171-174.

Steriade M, Nuñez A, Amzica F (1993) A novel slow ( $<1 \mathrm{~Hz}$ ) oscillation of neocortical neurons in vivo: depolarizing and hyperpolarizing components. J Neurosci 13:3252-3265.

Tierney PL, Dégenètais E, Thierry AM, Glowinski J, Gioanni Y (2004) Influence of the hippocampus on interneurons of the rat prefrontal cortex. Eur J Neurosci 20:514-524.

Tiesinga P, Sejnowski TJ (2009) Cortical enlightenment: are attentional gamma oscillations driven by ING or PING? Neuron 63:727-732.

Tseng KY, Kasanetz F, Kargieman L, Riquelme LA, Murer MG (2001) Cortical slow oscillatory activity is reflected in the membrane potential and spike trains of striatal neurons in rats with chronic nigrostriatal lesions. J Neurosci 21:6430-6439. 
Tseng KY, Mallet N, Toreson KL, Le Moine C, Gonon F, O’Donnell P (2006) Excitatory response of prefrontal cortical fast-spiking interneurons to ventral tegmental area stimulation in vivo. Synapse 59:412-417.

Vigneswaran G, Kraskov A, Lemon RN (2011) Large identified pyramidal cells in macaque motor and premotor cortex exhibit "thin spikes": implications for cell type classification. J Neurosci 31:14235-14242.

Walters JR, Hu D, Itoga CA, Parr-Brownlie LC, Bergstrom DA (2007) Phase relationships support a role for coordinated activity in the indirect pathway in organizing slow oscillations in basal ganglia output after loss of dopamine. Neuroscience 144:762-776.

Weinberger M, Mahant N, Hutchison WD, Lozano AM, Moro E, Hodaie M, Lang AE, Dostrovsky JO (2006) Beta oscillatory activity in the subthalamic nucleus and its relation to dopaminergic response in Parkinson's disease. J Neurophysiol 96:3248-3256.

Williams D, Tijssen M, Van Bruggen G, Bosch A, Insola A, Di Lazzaro
V, Mazzone P, Oliviero A, Quartarone A, Speelman H, Brown P (2002) Dopamine-dependent changes in the functional connectivity between basal ganglia and cerebral cortex in humans. Brain 125: $1558-1569$.

Wilson FA, O'Scalaidhe SP, Goldman-Rakic PS (1994) Functional synergism between putative gamma-aminobutyrate-containing neurons and pyramidal neurons in prefrontal cortex. Proc Natl Acad Sci U S A 91:4009-4013.

Zaidel A, Spivak A, Grieb B, Bergman H, Israel Z (2010) Subthalamic span of beta oscillations predicts deep brain stimulation efficacy for patients with Parkinson's disease. Brain 133:2007-2021.

Zold CL, Kasanetz F, Pomata PE, Belluscio MA, Escande MV, Galinanes GL, Riquelme LA, Murer MG (2012) Striatal gating through up states and oscillations in the basal ganglia: implications for Parkinson's disease. J Physiol Paris 106:40-46. 